O Mono Lake Water (Council and Bennett, 1993)

$\ominus$ Mono Lake Water (Bischoff et al., 1993)

$\Delta$ Mono Lake Shoreline Spring-water (Council and Bennett, 1993)

$\triangle$ Mono Lake Shoreline Spring-water (Bischoff et al., 1993)

$\diamond$ Big Soda Lake Water Above Chemocline (Kharaka et al., 1984)

$\ominus$ Big Soda Lake Water Below Chemocline (Kharaka et al., 1984)

$\uparrow$ Big Soda Lake Water (Clarke and Chatard, 1884)

$\square$ Big Soda Lake Ground-water (Kharaka et al., 1984)

\Shallow Ground-water Wells Near Big Soda Lake (Whitney, 1994)

Big Soda Lake Tufa Ground-water (this study)

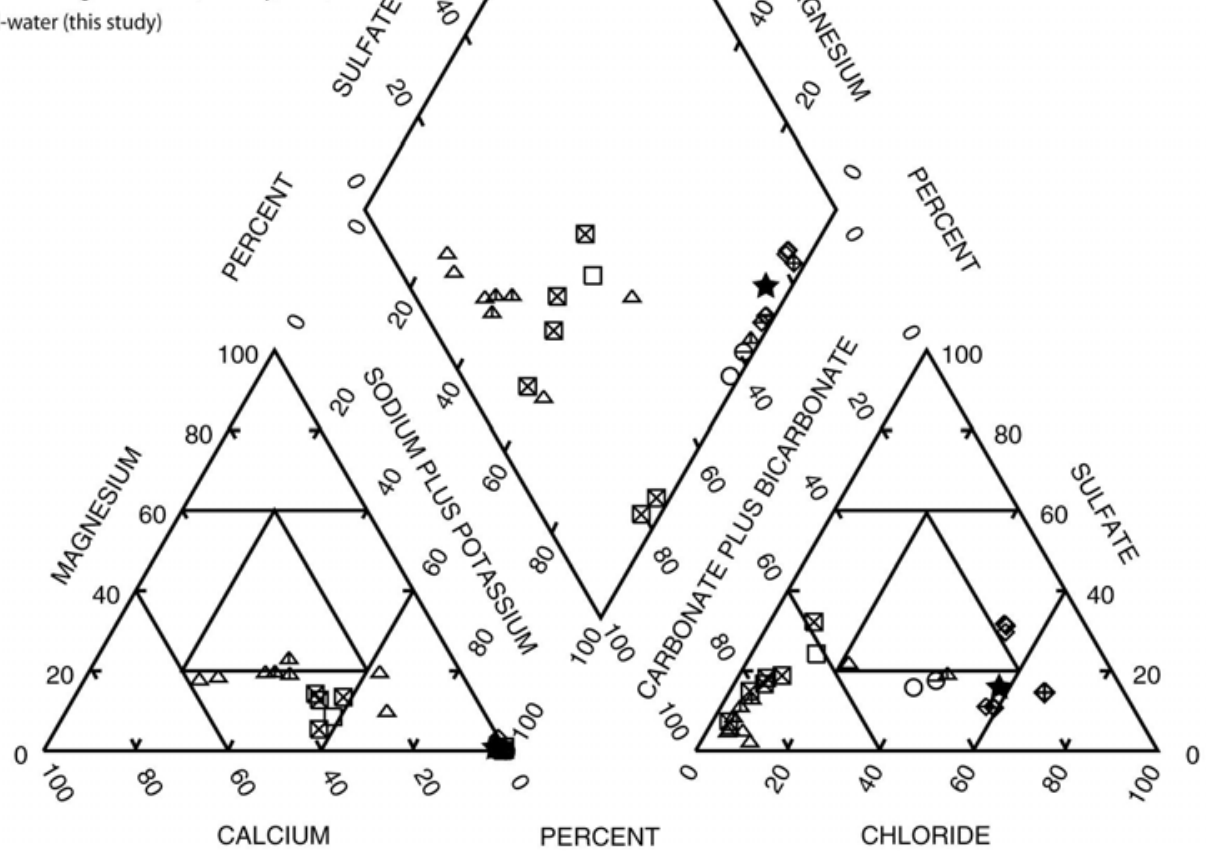

Figure DR1. Piper plot of the chemical composition of Big Soda Lake, regional ground water, and water discharging from the tufa mounds, compared to the water chemistry of Mono Lake and Mono Lake ground water.

\title{
References for figure:
}

Bischoff, J.L., Stine, S., Rosenbauer, R.J., Fitzpatrick, J.A., and Stafford, T.W., Jr., 1993, Ikaite precipitation by mixing of shoreline springs and lake water, Mono Lake, California, USA: Geochimica et Cosmochimica Acta, v. 57, p. 3855-3865.

Clarke, F.W., and Chatard, T.M., 1884, A report of work done in the Washington Laboratory during the fiscal year 1883-'84, Bulletin No. 9 of the United States Geological Survey: Washington, Government Printing Office, p. 39.

Council, T.C., and Bennett, P.C., 1993, Geochemistry of ikaite formation at Mono Lake, 
California: Implications for the origin of tufa mounds: Geology, v. 21, p. 971-974. Kharaka, Y.K., Robinson, S.W., Law, L.M., and Carothers, W.W., 1984,

Hydrogeochemistry of Big Soda Lake, Nevada; an alkaline meromictic desert lake: Geochimica et Cosmochimica Acta, v. 48, p. 823-835.

Whitney, R., 1994, Data on ground-water quality in the Carson River Basin, western Nevada and eastern California, 1987-90, U.S. Geological Survey Open-File Report 94-39, p. 139.

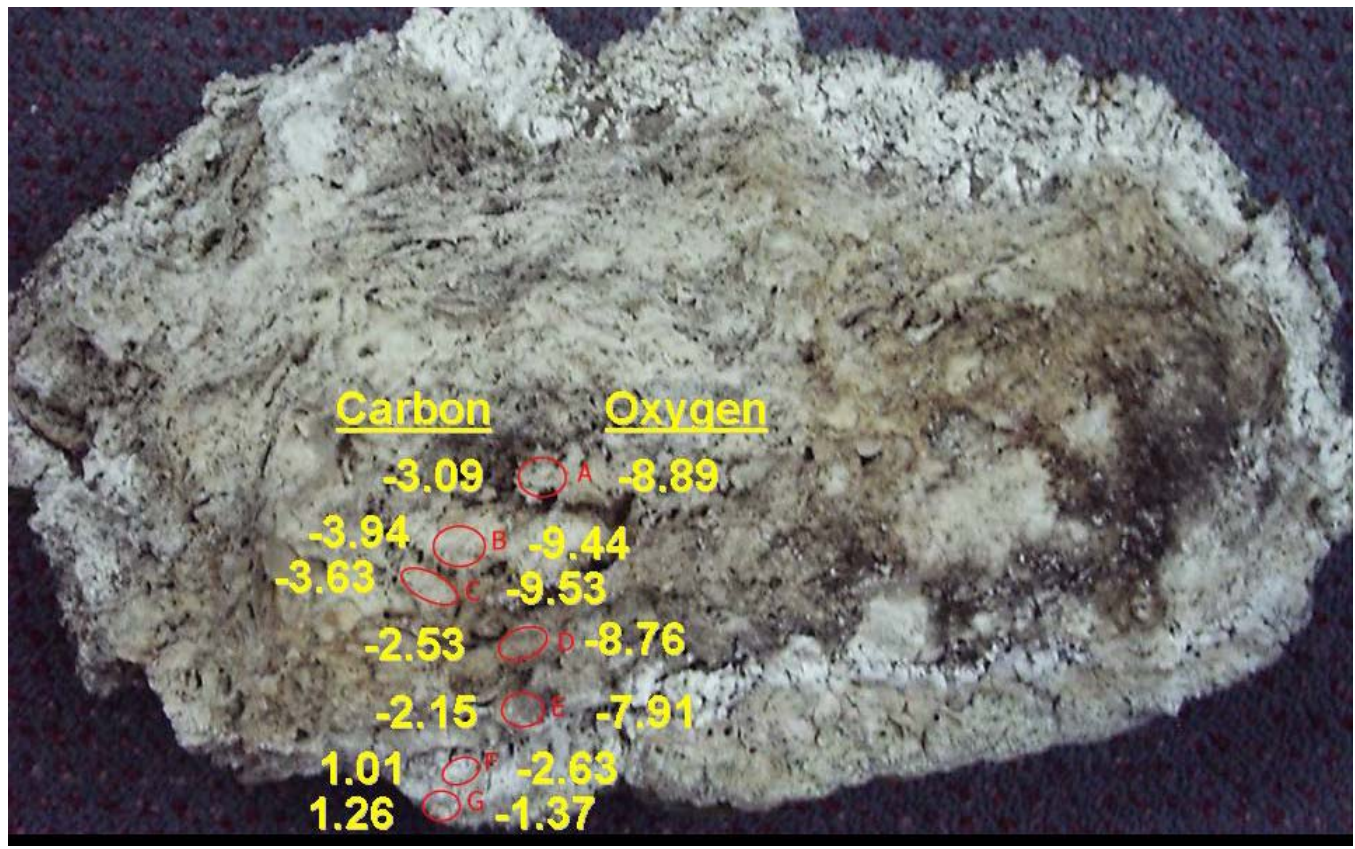

Figure DR2. Photograph of S1 showing the oxygen and carbon isotope values from the center to the outside of the tufa mound. Values at site $\mathrm{E}$ are an average of two samples. The sample is approximately $0.2 \mathrm{~m}$ in diameter. 
Table DR1. Water chemistry data for Mono Lake, Big Soda Lake and Big Soda Lake ground water. Data in milliequivalents per liter

\begin{tabular}{|c|c|c|c|c|c|c|}
\hline Location & $\mathrm{Ca}$ & $\mathrm{Mg}$ & $\mathrm{Na}+\mathrm{K}$ & $\mathrm{Cl}$ & $\mathrm{HCO}_{3}$ & $\mathrm{SO}_{4}$ \\
\hline \multicolumn{7}{|l|}{ Mono Lake from Council and Bennett, $1993^{1}$. } \\
\hline Mono Lake & 0.05 & 2.64 & 1462 & 685 & 618 & 277 \\
\hline Nearshore spring ML S1 & 6.90 & 3.50 & 8.09 & 1.07 & 14.90 & 2.33 \\
\hline Nearshore spring ML S2 & 2.33 & 1.49 & 2.73 & 0.30 & 6.40 & 0.60 \\
\hline Nearshore spring ML S3 & 7.74 & 3.72 & 7.75 & 0.85 & 16.60 & 2.11 \\
\hline \multicolumn{7}{|l|}{ Mono Lake from Bischoff et al., $1993^{2}$. } \\
\hline Mono Lake & 0.20 & 2.71 & 1377 & 518 & 593 & 208 \\
\hline Nearshore spring ML S1B & 6.74 & 2.06 & 2.93 & 0.51 & 9.41 & 0.54 \\
\hline Nearshore spring ML S2B & 5.54 & 1.89 & 2.99 & 0.48 & 9.28 & 0.46 \\
\hline Nearshore spring ML S3B & 6.69 & 3.04 & 6.05 & 1.24 & 13.85 & 0.75 \\
\hline Nearshore spring ML S4B & 8.43 & 3.87 & 27.97 & 8.35 & 20.98 & 8.06 \\
\hline Nearshore spring ML S4 & 0.05 & 2.49 & 1109 & 509 & 407 & 214 \\
\hline Thermal Spring NBHS & 6.24 & 6.83 & 22.27 & 3.84 & 30.99 & 0.71 \\
\hline \multicolumn{7}{|l|}{ Big Soda Lake from Kharaka et al., $1984^{3}$. } \\
\hline Surface & 0.25 & 12.17 & 361.8 & 194.6 & 66.5 & 121.0 \\
\hline 10 meters below surface & 0.25 & 12.26 & 361.8 & 199.1 & 66.5 & 121.4 \\
\hline 20 meters below surface & 0.24 & 12.17 & 359.5 & 199.7 & 66.5 & 120.1 \\
\hline 30 meters below surface & 0.23 & 12.01 & 368.4 & 204.2 & 70.8 & 115.8 \\
\hline 35 meters below surface & 0.04 & 1.08 & 1284.7 & 705.2 & 390.1 & 135.1 \\
\hline 40 meters below surface & 0.04 & 0.46 & 1204.6 & 772.9 & 395.0 & 145.5 \\
\hline 50 meters below surface & 0.04 & 0.45 & 1195.7 & 784.1 & 395.0 & 142.2 \\
\hline 60 meters below surface & 0.04 & 0.47 & 1196.2 & 778.5 & 395.0 & 139.5 \\
\hline Surface water data from Clarke and Chatard, $1884^{4}$. & No Data & 22.2 & 2058.4 & 1288.7 & 333.2 & 269.8 \\
\hline $\begin{array}{l}\text { Tufa spring water (USGS unpublished data) } \\
\text { Regional ground water from Big Soda Lake area (number }\end{array}$ & 0.19 & 0.12 & 15.2 & 9.0 & 4.0 & 2.3 \\
\hline \multicolumn{7}{|l|}{$\begin{array}{l}\text { is USGS well ID) from Whitney, } 1994^{5} \text { and Allander et al, } \\
2001^{6} \text {. }\end{array}$} \\
\hline 392903118524401 & 3.54 & 1.48 & 5.37 & 0.99 & 6.03 & 3.33 \\
\hline 392926118533001 & 2.64 & 0.99 & 4.15 & 0.71 & 5.41 & 1.42 \\
\hline 393004118511301 & 0.14 & 0.05 & 8.91 & 0.39 & 7.31 & 1.35 \\
\hline 393004118514201 & 0.92 & 0.43 & 1.88 & 0.38 & 9.51 & 0.78 \\
\hline 393006118515101 & 0.03 & 0.01 & 4.17 & 0.25 & 3.00 & 0.72 \\
\hline
\end{tabular}


393038118512201

${ }^{1}$ Council, T.C., and Bennett, P.C., 1993, Geochemistry of ikaite formation at Mono Lake, California: Implications for the origin of tufa mounds: Geology, v. 21, p. 971-974.

${ }^{2}$ Bischoff, J.L., Stine, S., Rosenbauer, R.J., Fitzpatrick, J.A., and Stafford, T.W., Jr., 1993, Ikaite precipitation by mixing of shoreline springs and lake water, Mono Lake, California, USA: Geochimica et Cosmochimica Acta, v. 57, p. 3855-3865.

${ }^{3}$ Kharaka, Y.K., Robinson, S.W., Law, L.M., and Carothers, W.W., 1984, Hydrogeochemistry of Big Soda Lake, Nevada; an alkaline meromictic desert lake: Geochimica et

Cosmochimica Acta, v. 48, p. 823-835.

${ }^{4}$ Clarke, F.W., and Chatard, T.M., 1884, A report of work done in the Washington Laboratory during the fiscal year 1883-'84, Bulletin No. 9 of the United States Geological Survey: Washington, Government Printing Office, 39 p.

${ }^{5}$ Whitney, R., 1994, Data on ground-water quality in the Carson River Basin, western Nevada and eastern California, 1987-90, U.S. Geological Survey Open-File Report 94-39, p. 139.

${ }^{6}$ Allander, K., Gortsema, G., Hutchinson, D., and

Schwartzenberger, J., 2001, Water resources data -- Nevada -Water year 2000, U.S. Geological Survey Water Data Report NV-00-1, $570 \mathrm{p}$. 
Table DR2. Stable isotope analyses and mineral identification

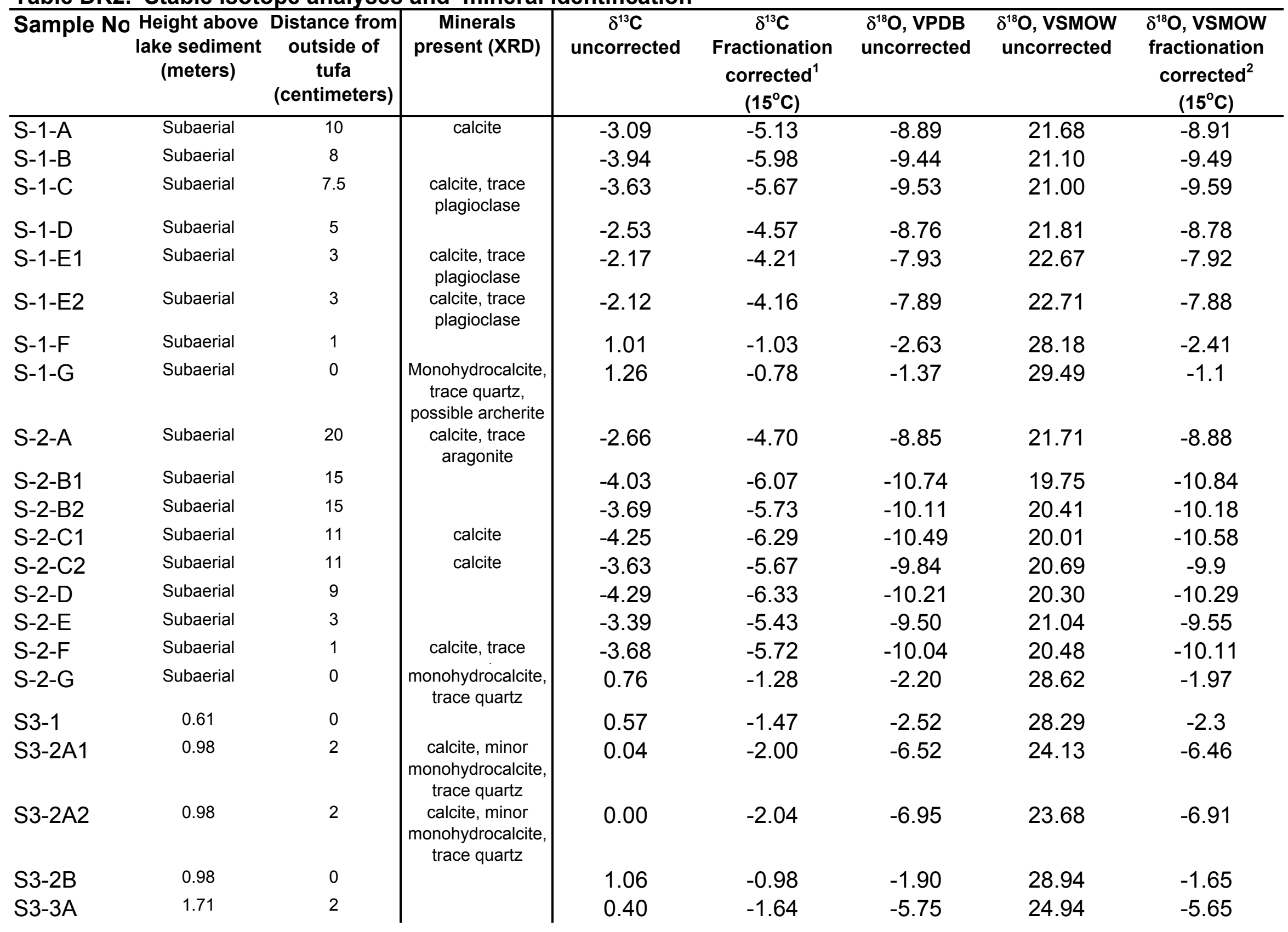




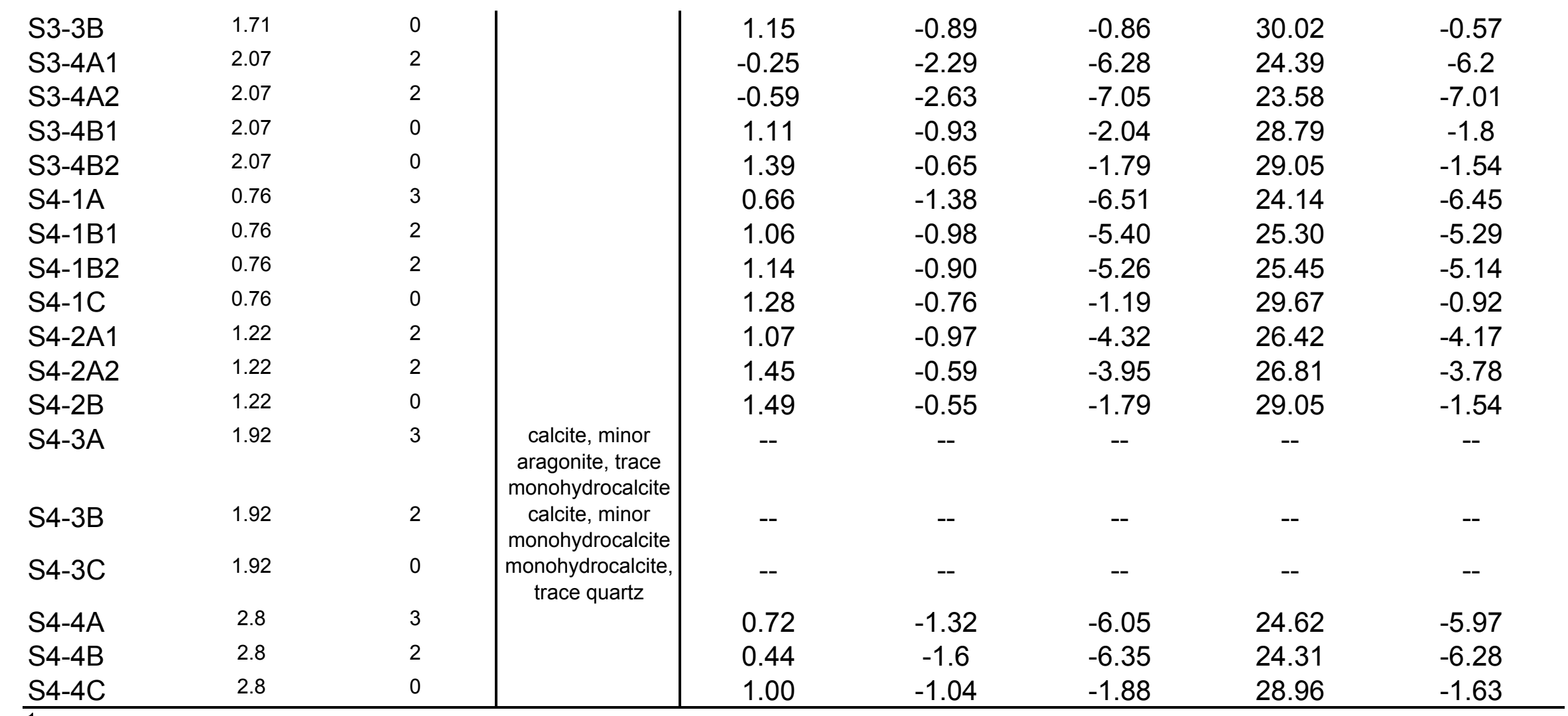

${ }^{1}$ Fractionation factor for carbon from Deines et al, 1974.

${ }^{2}$ Fractionation factor for oxygen from O'Neil et al, 1969. 